\title{
Decanoic acid attenuates the excitability of nociceptive trigeminal primary and secondary neurons associated with hypoalgesia
}

This article was published in the following Dove Press journal: Journal of Pain Research

\author{
Ryousuke Nakajima' \\ Airi Uehara' \\ Shiori Takehana' \\ Youichi Akama² \\ Yoshihito Shimazu' \\ Mamoru Takeda' \\ 'Laboratory of Food and Physiological \\ Sciences, Department of Life and \\ Food Sciences, School of Life and \\ Environmental Sciences, Azabu \\ University, Chuo-ku, Sagamihara, \\ Kanagawa 252-520I, Japan; \\ ${ }^{2}$ Department of Emergency, Minami \\ Touhoku Hospital, Iwanuma, Miyagi \\ 989-2483, Japan
}

Background: Acute application of decanoic acid (DA) in vivo suppresses the excitability of spinal trigeminal nucleus caudalis ( $\mathrm{SpVc}$ ) wide dynamic range (WDR) neurons associated with the short-term mechanical hypoalgesia via muscarinic $\mathrm{M}_{2}$ receptor signaling; however, the effect of DA on nociceptive trigeminal ganglion (TG) and SpVc nociceptive-specific (NS) neuronal excitability under in vivo conditions remains to be determined. The present study investigated whether this effect could be observed in naive rats.

Results: Extracellular single-unit recordings were made from TG and $\mathrm{SpVc}$ NS neurons of pentobarbital-anesthetized rats in response to orofacial noxious mechanical stimuli. DA inhibited the mean firing frequency of both TG and SpVc NS neurons, reaching a maximum inhibition of discharge frequency within 1-5 minutes and reversing after approximately 10-minutes; however, this DA-induced suppression of SpVc NS neuronal firing frequency did not occur in rats administered with methoctramine intravenously prior to stimulation.

Conclusion: This in vivo study indicated that firing of TG and SpVc NS neurons induced by mechanical hypoalgesia through peripheral $\mathrm{M}_{2}$ receptors could be inhibited by acutely administered DA, implicating the potential of DA in the future treatment of trigeminal pain.

Perspective: This article presents that the acute DA application suppresses the excitability of TG and SpVc NS neurons associated with mechanical hypoalgesia via peripheral $\mathrm{M}_{2}$ receptor signaling, supporting DA as a potential therapeutic agent in complementary and alternative medicine for the attenuation of nociception.

Keywords: decanoic acid, trigeminal spinal nucleus, nociception, hypoalgesia, single-unit recording, complementary alternative medicine

\section{Introduction}

Nociceptive sensory data derived from trigeminal ganglion (TG)-innervated orofacial regions that connect spinal trigeminal nucleus caudalis $(\mathrm{SpVc})$ neurons and the upper cervical $(\mathrm{C} 1-\mathrm{C} 2)$ dorsal horn provide critical conduits for trigeminal nociceptive pain associated with inflammation and tissue injury. ${ }^{1-3} \mathrm{SpVc}$ nociceptive neurons were classified as nociceptive specific (NS) and wide dynamic range (WDR) based on their sensitivity to mechanical stimulation applied to the orofacial area such as facial skin. WDR neurons responded to both noxious and nonnoxious stimulations. ${ }^{3}$ Since graded noxious stimuli applied to receptive field produce increased firing frequency of $\mathrm{SpVc}$ WDR neurons in proportion to stimulus intensity, it can be assumed that WDR neurons play an important role in encoding stimulus intensity. However, NS neurons respond only to noxious stimulation of receptive field and therefore play an important
Correspondence: Mamoru Takeda Laboratory of Food and Physiological Sciences, Department of Life and Food Sciences, School of Life and Environmental Sciences, Azabu University, I-17-7I, Fuchinobe, Chuo-ku, Sagamihara, Kanagawa 252-520I, Japan

$\mathrm{Tel}+8 \mid 42769$ I886

Fax +81427692212

Email m-takeda@azabu-u.ac.jp 
role in encoding noxious stimulus localization via nociceptive TG neurons to higher centers in the central nervous system.

Complementary and alternative medicine (CAM), such as herbal medicines and acupuncture, has been trialed for treating persistent clinical chronic pain. ${ }^{4-6}$ For example, the potential effects of diet and dietary supplementation on conditions associated with pain have been the focus of considerable research. ${ }^{7-9}$ Indeed, polyphenolic compounds (eg, resveratrol and chlorogenic acid) and fatty acids (eg, decanoic acid [DA] and docosahexaenoic acid) modulate the neuronal excitability of the peripheral nerves, including those of the sensory system, via various voltage-dependent ionic channels, ligand-gated channels, and neurotransmitter receptors. ${ }^{10-13}$ As previously discussed, DA displays several potential biological activities, including the antagonism of muscarinic acetylcholine receptors (mAchRs). ${ }^{14}$ Indeed, we previously showed that DA applied acutely as an ointment induces mechanical hypoalgesia, predominantly by limiting the SpVc WDR neuronal excitability via peripheral acetylcholine muscarinic $2\left(\mathrm{M}_{2}\right)$ receptors, suggesting a role of this fatty acid in CAM for trigeminal nociceptive pain present without inflammation or neuropathy. ${ }^{11}$ Thus, the present study further investigated how a similar acute treatment with DA affects TG and SpVc NS neuronal activities associated with hypoalgesia in naive rats.

\section{Methods}

The Animal Use and Care Committee of Azabu University approved the present study. The protocols also adhered to the ethical guidelines of the International Association for the Study of Pain; ${ }^{15}$ specifically, we used as few animals as possible to achieve the target results and minimized their suffering wherever possible.

\section{Local, cutaneous application of DA as an ointment}

DA (molecular weight $[\mathrm{MW}]=172.26 \mathrm{kDa}$ ) is a saturated, medium-chain fatty acid with a 10-carbon backbone $\left(\mathrm{C}_{10} \mathrm{H}_{20} \mathrm{O}_{2}\right)$ and is lipophilic (log $\left.P=4.09\right)$. Therefore, we formulated an ointment for in vivo application of DA (Patent number: P2013-139421A, Japan), as described and defined previously. ${ }^{11}$

\section{Electrophysiological experiments for single-unit recording of neurons}

Electrophysiological recordings were conducted on 26 adult male Wistar rats weighing 230-310 g (18 rats: SpVc NS neurons; 8 rats: TG neurons), as previously described. ${ }^{11}$ In brief, after anesthesia and maintenance with pentobarbital sodium through a cannula into the jugular veins, animals were placed in a stereotaxic apparatus for extracellular activity recordings of single SpVc neurons, according to the coordinates demonstrated by Paxinos and Watson, ${ }^{16}$ using a glass micropipette filled with $2 \%$ pontamine sky blue and $0.5 \mathrm{M}$ sodium acetate. ${ }^{11,17}$ For recordings of single TG neurons, the rats underwent a craniotomy and hemispherectomy to expose the trigeminal ganglia, and the activity was recorded from the left side of the neurons, as described for the SpVc units. ${ }^{18,19}$ All neuronal recordings were amplified (DAM80; World Precision Instruments, Sarasota, FL, USA), filtered (0.3-10 KHz), monitored with an oscilloscope (SS-7672; Iwatsu, Tokyo Japan), and then recorded for offline analysis using Power Lab and Chart 5 software (ADInstruments Ltd, Oxford, UK), as described previously. ${ }^{11,17}$ During all recordings, we monitored anesthesia state of the rats based on the absence of corneal reflex and response to paw pinching as well as rectal temperatures, and wound margins were continuously covered with a local anesthetic, $2 \%$ lidocaine.

\section{Experimental protocols}

We conducted extracellular recordings of SpVc NS and TG neuronal activities as previously described. ${ }^{11,17}$ Briefly, we immediately identified the receptive field on the left side of the orofacial skin (whisker pad) using mechanical stimulation without sensitizing the peripheral receptors. We then applied noxious pinch and mechanical stimulation to the whisker pad using von Frey hairs (>15 g: 15, 26, and $60 \mathrm{~g}$ ) and forceps, indentified the responsive SpVc WDR neurons, and tested for spontaneous discharge. Subsequently, we determined the threshold for mechanical nociception using nonnoxious and noxious stimulation with von Frey hairs (4, 6, 10, 15, 26 , and $60 \mathrm{~g}$ ) applied at 5-second intervals. The mechanical receptive field of neurons was mapped by probing the facial skin with von Frey hairs and then outlined on a life-sized drawing of a rat on tracing paper. Mechanically induced TG and SpVc NS neuronal discharges were quantified, and spontaneous discharge frequencies were determined over 2-5 minutes, with no discharge indicating a "silent" neuron. We then compared the mean neuronal firing rates following mechanical stimulation before and after drug administration and generated poststimulus histograms (bin=100 ms). SpVc NS neuronal activity following DA and vehicle treatments was evaluated during the peak effect and recovery period at $1,3,10$, and 20 minutes. In addition, we assessed DAinduced changes in firing of the mechanically stimulated $\mathrm{SpVc}$ WDR neurons based on the effect of pretreatment with an $\mathrm{M}_{2} \mathrm{mAchR}$-specific antagonist, methoctramine (1 mM; 
Sigma-Aldrich Co., St Louis, MO, USA) 10 minutes before the DA treatment $(\mathrm{n}=6) .{ }^{11}$

\section{Identification of recording sites for SpVc NS neurons}

At the end of the recording sessions, rats were deeply anesthetized, and anodal DC currents were passed through the recording micropipettes ( $30 \mu \mathrm{A}, 5$ minutes), followed by transcardial perfusion with $10 \%$ formalin in saline. Coronal sections were cut frozen $(30-\mu \mathrm{m}$ thickness) for H\&E staining. Recording sites were identified from the blue spots, and electrode tracks were constructed based on the micromanipulator readings.

\section{Data analyses}

Values are expressed as mean \pm standard error of the mean. Statistical analyses were performed using one-way repeated measures ANOVA, followed by Tukey-Kramer/Dunnett tests (post hoc test) for behavioral and electrophysiological data. $P<0.05$ was considered statistically significant.

\section{Results}

\section{General properties of TG neurons} responding to mechanical stimulation of orofacial skin

Extracellular single-unit activity was recorded from eight neurons in the trigeminal ganglia. The TG neurons responding to noxious mechanical stimulation exhibited a somatic receptive field in the orofacial area (mainly whisker pad; Figure 1A), as described previously. ${ }^{18,19}$ No units exhibited spontaneous discharges. As shown in Figure 1B, recording sites were found in the mandibular branches, as described previously ${ }^{20}$ and typical examples of nociceptive TG neuronal unit responses are shown in Figure 1C. Graded mechanical
A

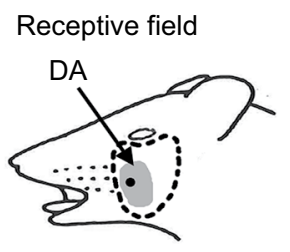

B

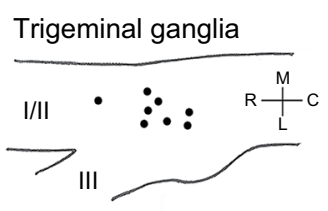

C
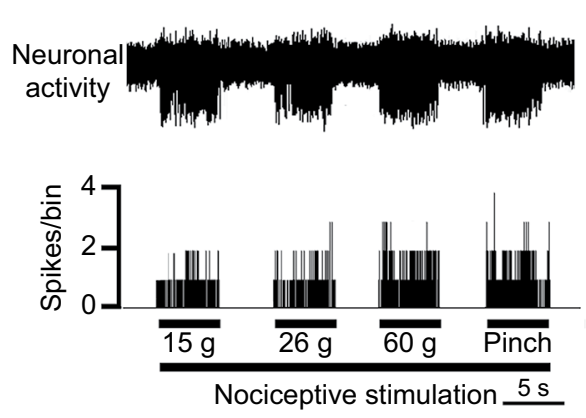

D

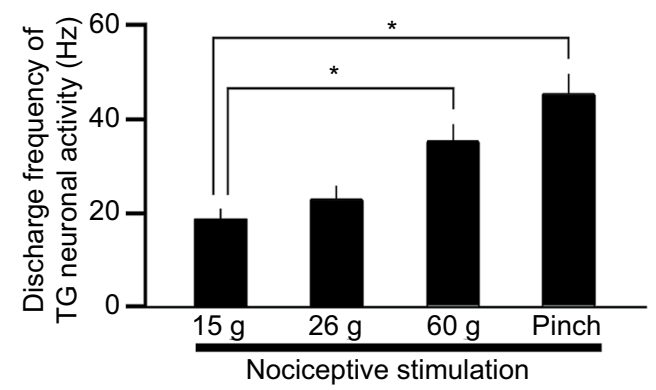

E

Before

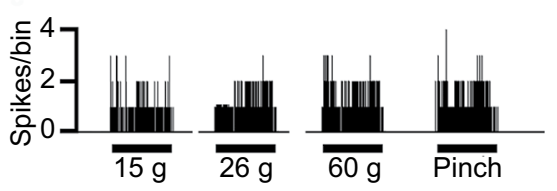

After DA $\stackrel{\subseteq}{\stackrel{0}{0}}]$ application 2 (1 $\mathrm{min})$
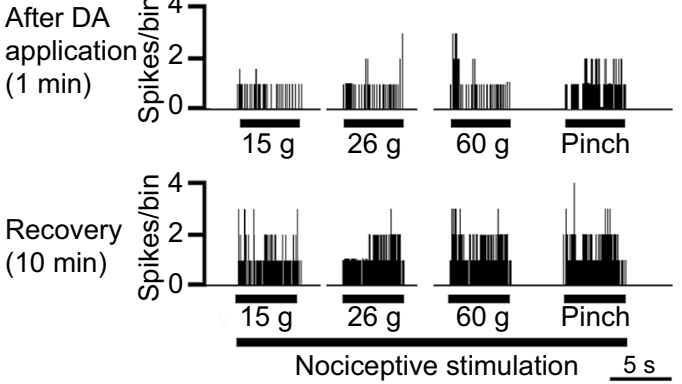

$\mathbf{F}$

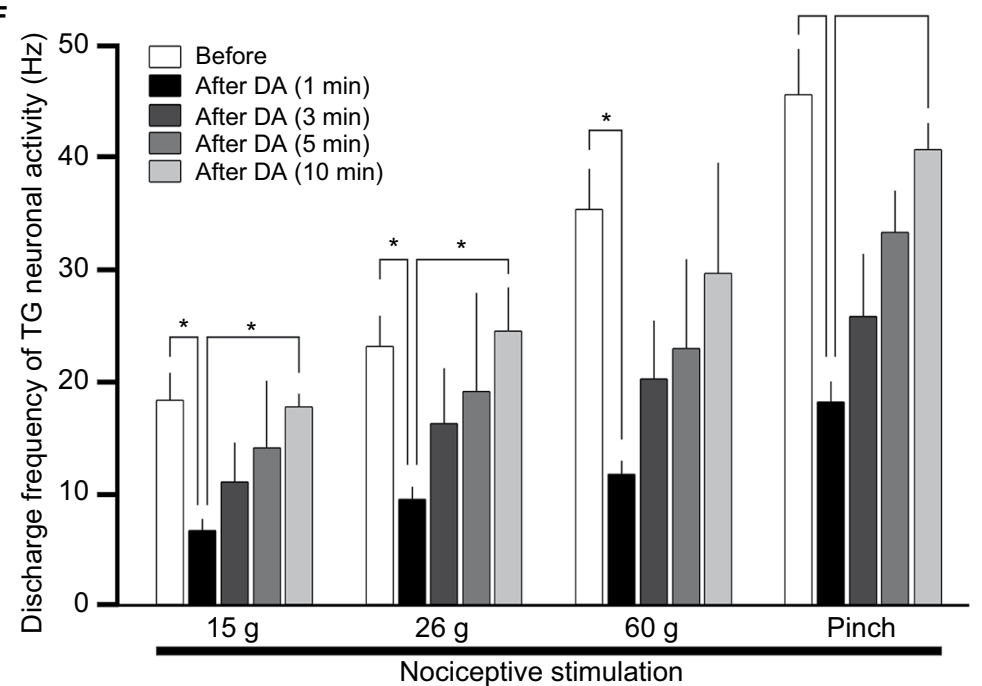

Figure I Effect of DA treatment on TG neuronal activity in response to mechanical stimulation of orofacial skin.

Notes: (A-D) General characteristics of the TG neuronal activity in response to mechanical stimulation of orofacial skin. (A) Typical example of the receptive field of a whisker pad in the facial skin; the shaded area indicates the region treated with DA. (B) Distribution of NS neurons responding to noxious mechanical stimulation of the facial skin ( $n=8)$. (C) Example of noxious mechanical stimulation-induced firing of TG neurons. (D) Summary of noxious mechanical stimulation-induced TG neuronal discharge frequency. (E) Typical example of noxious $(15,26$, and $60 \mathrm{~g})$ mechanical and noxious pinch stimulation-evoked TG neuron activity before DA treatment, I minute after DA treatment, and 10 minutes after DA treatment. (F) Time course of the effects of DA treatment on the mean firing frequency of TG neurons in response to mechanical stimulation of orofacial skin. ${ }^{*} P<0.05$; before DA treatment vs $I, 3,5$, and 10 minutes after DA treatment.

Abbreviations: DA, decanoic acid; NS, nociceptive specific; TG, trigeminal ganglion. 
stimulation was applied to the most sensitive area of the receptive field, which exhibited increased firing frequency of TG neurons in proportion to stimulus intensity (Figure 1D). The mean mechanical stimulation-induced spike threshold was $11.5 \pm 2.8 \mathrm{~g}(\mathrm{n}=6)$. Every neuron recorded belonged to the category of NS neurons.

\section{Effect of DA on the excitability of TG neurons responding to nonnoxious and noxious mechanical stimulation}

Figure 1E shows typical examples of DA treatment effects on the excitability of TG neurons in response to noxious mechanical and pinch stimulation, as described in earlier studies. ${ }^{11}$ Briefly, these effects constituted inhibition of TG neuronal activity 1-5 minutes after DA treatment with a return to control levels in approximately 10 minutes for both modes of noxious stimulations. As indicated in Figure 1F, the mean firing rates of stimulated TG neurons decreased significantly after DA treatment, compared with rates before DA treatment ( $15 \mathrm{~g}$ stimulus, $18.1 \pm 1.3 \mathrm{vs} 7.3 \pm 0.5$ $\mathrm{Hz}, F=10.3$, n=8; $60 \mathrm{~g}$ stimulus, $35.2 \pm 2.2$ vs $12.1 \pm 0.6 \mathrm{~Hz}$, $F=4.1, \mathrm{n}=6$; and pinch stimulus, $46.1 \pm 2.2$ vs $18.5 \pm 1.1 \mathrm{~Hz}$, $F=7.2, \mathrm{n}=8$, before vs after DA for 3 minutes, respectively). No significant changes were observed in the mean receptive field size after DA administration $(11.5 \pm 0.1$ vs $10.5 \pm 0.3$ $\mathrm{mm}^{2}$, before vs after DA, respectively) or in the spontaneous firing rate. Vehicle treatment had no significant effect on either spontaneous or evoked (noxious mechanical and pinch stimulation) activity of the TG neurons $(n=4$; data not shown).

\section{General properties of SpVc NS neurons responding to mechanical stimulation of orofacial skin}

Next, we observed inhibited firing in all 10 SpVc NS neurons tested for their responses to DA following nonnoxious and noxious mechanical stimulations of the whisker pad. Of these, three neurons did not recover their control levels of activity, leaving seven SpVc NS neurons for further analyses. Confirming our previous findings, these responsive neurons displayed a somatic receptive field in the buccal region of the orofacial skin (Figure 2A), with recording sites mainly distributed in the maxillary and mandibular branches in layers I-II $(n=7)$ and IV $(n=3)$ of the SpVc (obex, -1.0 to $-2.0 \mathrm{~mm}$; Figure 2B). ${ }^{11}$ The inset in Figure $2 \mathrm{~B}$ represents the histological confirmation conducted for each such recording site. Finally, we identified the most sensitive area of the receptive field with respect to the stimulus-proportional increase in firing frequency of SpVc NS neurons induced by the graded mechanical stimulation (Figure 2C).
A Receptive field

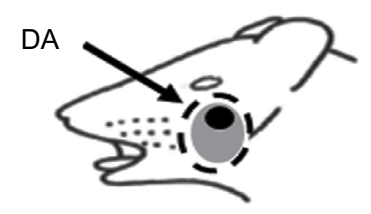

C SpVcNS neuron
Wave form of action potential

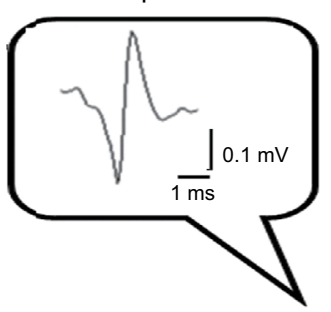

B SpVc
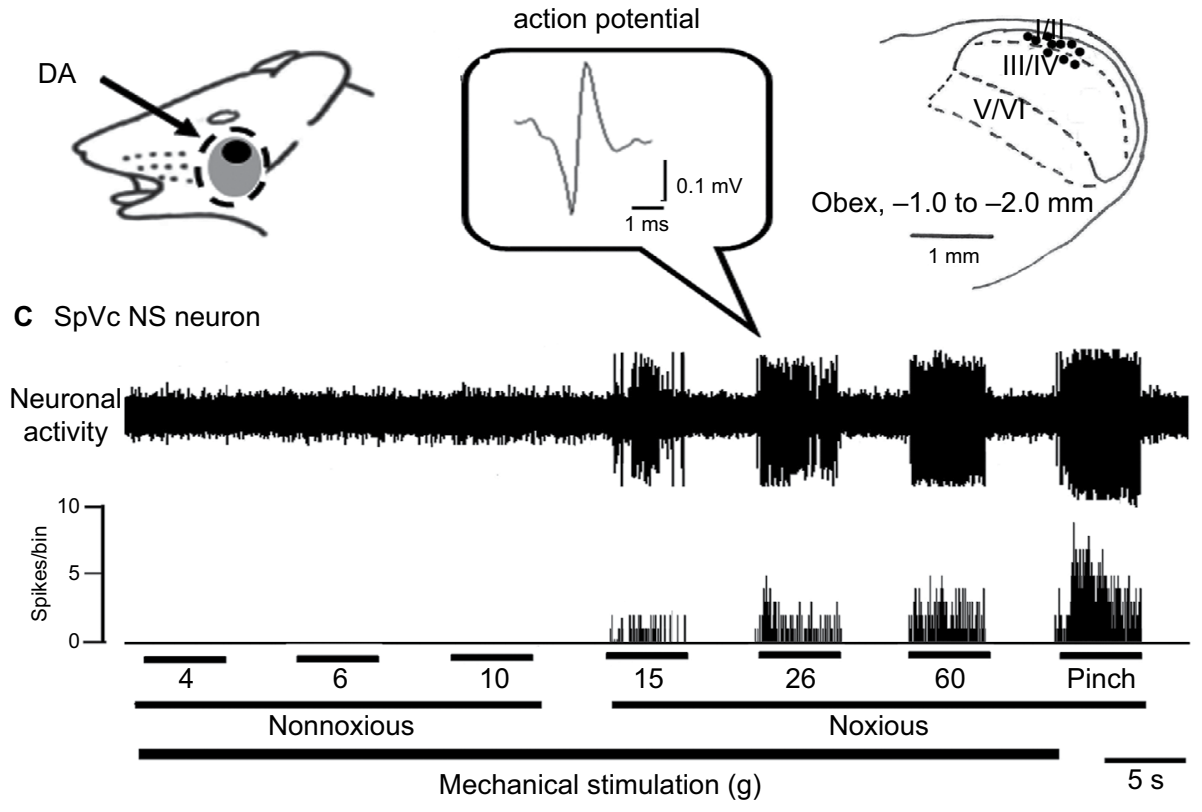

Figure 2 General characteristics of SpVc NS neuronal activity in response to mechanical stimulation of the orofacial skin.

Notes: (A) Typical example of receptive field of whisker pad in the facial skin. Shaded area indicates the region treated with DA. (B) Distribution of SpVc NS neurons responding to noxious mechanical stimulation of the facial skin $(n=10)$. Inset, waveform of action potential. The number below each drawing indicates the frontal plane in relation to the obex. (C) Example of noxious mechanical stimulation-induced firing of SpVc NS neurons.

Abbreviations: DA, decanoic acid; NS, nociceptive specific; SpVc, spinal trigeminal nucleus caudalis. 


\section{Effect of DA on the excitability of SpVc NS neurons responding to noxious mechanical stimulation}

Figure 3 further represents the typical DA effects on $\mathrm{SpVc}$ NS neuronal excitability in response to noxious stimulation, with inhibition of the firing obvious from 1 to 5 minutes after DA treatment, and activity returning to control levels within 10 minutes. The mean SpVc NS neuronal firing rates induced by both mechanical and pinch stimulations decreased significantly after DA treatment (15 g stimulus, $15.7 \pm 1.5 \mathrm{vs}$

A
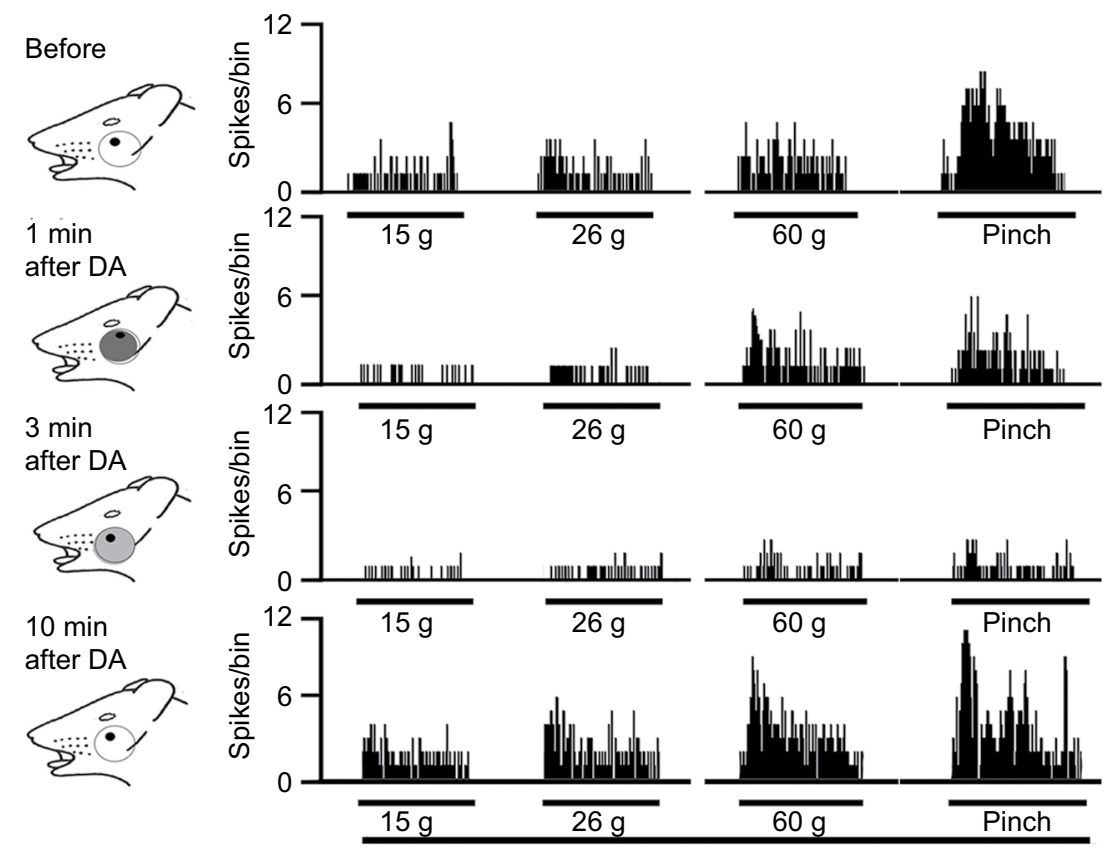

$3 \min$ after DA

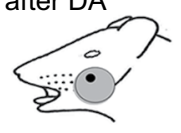

10 min
after DA

Noxious mechanical stimulation

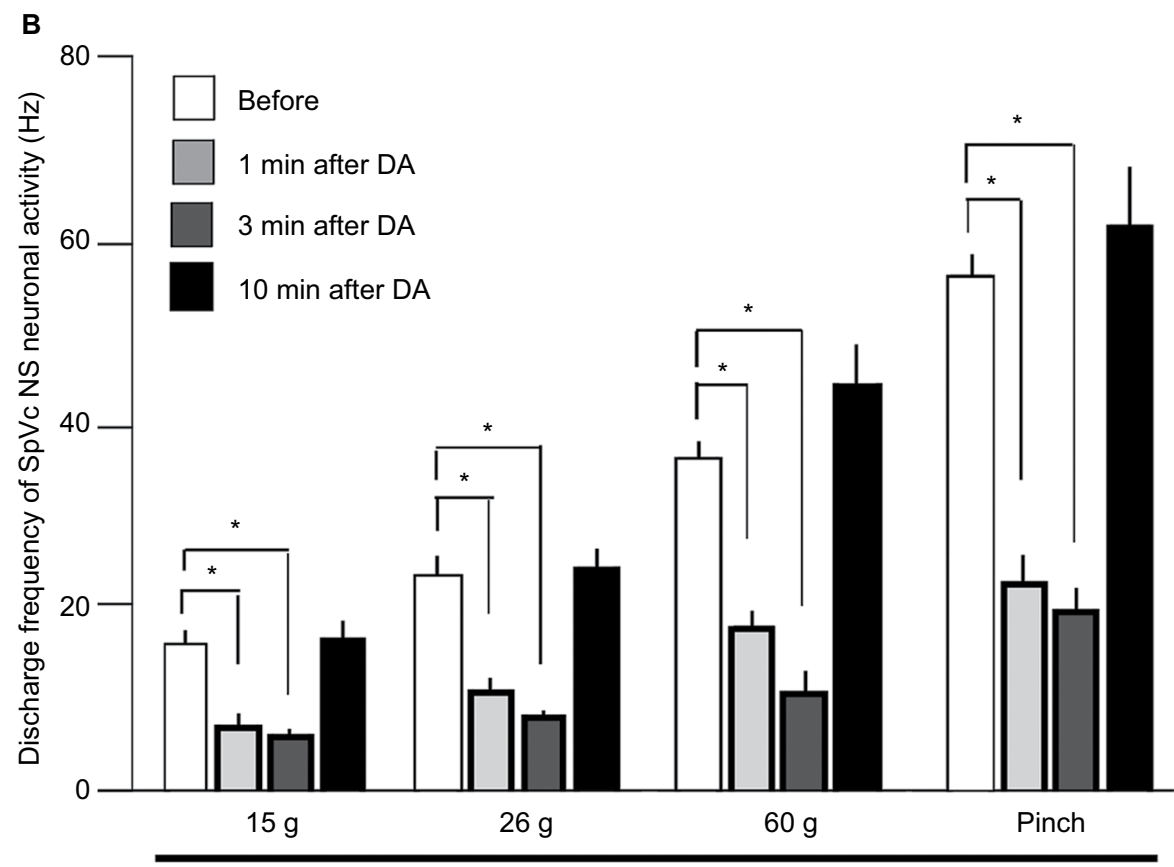

Noxious mechanical stimulation

Figure 3 Effect of DA treatment on SpVc NS neuronal activity in response to mechanical stimulation of the orofacial skin.

Notes: (A) Typical example of noxious (I5 and $60 \mathrm{~g}$ ) mechanical and noxious pinch stimulation-evoked SpVc WDR neuron activity before DA treatment and I, 3, 5, and 10 minutes after DA treatment. Receptive field of the whisker pad in the facial skin. Blackened area indicates the location and size of the receptive field. (B) Time course of the effects of DA treatment on the mean firing frequency of SpVc NS neurons in response to mechanical stimulation of the orofacial skin. $* P<0.05 ; 6 \mathrm{~g}$ vs 10 , 15 , and $60 \mathrm{~g}$ and pinch stimulation.

Abbreviations: DA, decanoic acid; NS, nociceptive specific; SpVc, spinal trigeminal nucleus caudalis; WDR, wide dynamic range. 
$5.5 \pm 0.7 \mathrm{~Hz}, F=13.6, \mathrm{n}=7 ; 60 \mathrm{~g}$ stimulus, $36.4 \pm 2.1$ vs $10.9 \pm 1.8$ $\mathrm{Hz}, F=35.0, \mathrm{n}=7$; and pinch stimulus, $57.1 \pm 3.3$ vs $19.4 \pm 2.3$ $\mathrm{Hz}, F=37.7, \mathrm{n}=7$, before DA treatment vs 3 minutes after DA treatment, respectively), but there were no significant changes in either mean receptive field size $(12.5 \pm 0.1$ vs $13.9 \pm 0.3$ $\mathrm{mm}^{2}$, before vs after DA, respectively) or spontaneous firing. Vehicle treatment had no significant effects on the activity of SpVc NS neurons ( $\mathrm{n}=2$; data not shown).

\section{Pretreatment with $M_{2} m A c h R$ antagonist attenuates the DA-induced inhibition of SpVc NS neurons responding to noxious stimulation}

Pretreatment with the $\mathrm{M}_{2} \mathrm{mAchR}$ antagonist methoctramine (1 mM, intravenously [iv]) significantly inhibited the DAinduced suppression of $\mathrm{SpVc}$ NS neuronal firing in response to noxious stimulation $(\mathrm{n}=6$; Figure $4 ; 15 \mathrm{~g}, 9.1 \pm 3.3$ vs $24.5 \pm 2.3 \mathrm{~Hz}, F=6.9, \mathrm{n}=6 ; 26 \mathrm{~g}, 12.4 \pm 2.0$ vs $33.1 \pm 2.5 \mathrm{~Hz}$, $F=9.2, \mathrm{n}=6 ; 60 \mathrm{~g}, 19.1 \pm 2.6$ vs $46.2 \pm 5.1, F=15.2, \mathrm{n}=6$; and pinch, $25.2 \pm 4.6$ vs $52.4 \pm 5.2 \mathrm{~Hz}, F=15.5, \mathrm{n}=6$, for DA vs DA+methoctramine, respectively).

\section{Comparison of DA-induced inhibition between SpVc NS and WDR neuronal activity}

Finally, we compared the relative inhibitory effect of DA on neuronal discharges of SpVc NS and WDR neurons using our published data. ${ }^{11}$ Although we previously observed a tendency that the magnitude of inhibition of SpVc NS neurons was stronger than those of SpVc WDR neurons, the mean magnitude of inhibition by DA of discharge frequency was not significantly greater for NS neurons compared to WDR neuron (NS vs WDR: $15 \mathrm{~g}, 63.5 \% \pm 5.8 \%$ vs $55.0 \% \pm 6.5 \%$; $26 \mathrm{~g}, 61.7 \% \pm 5.7 \%$ vs $51.0 \% \pm 5.6 \%$; $60 \mathrm{~g}, 67.6 \% \pm 6.1 \%$ vs $49.0 \% \pm 8.6 \%$; and pinch, $63.1 \% \pm 4.7 \%$ vs $53.0 \% \pm 7.0 \%$ ).

\section{Discussion}

\section{Inhibition of SpVc NS neuronal excitability by DA}

Noxious sensory information in the area innervating the trigeminal nerve is relayed from trigeminal primary afferents (TG neurons) to second-order neurons in the $\mathrm{SpVc}$ and the C1-C2 dorsal horn. ${ }^{1-3}$ Two types of nociceptive neurons exist in the $\mathrm{SpVc}$ based on their sensitivity to mechanical stimulation applied to the orofacial area, such as facial skin. ${ }^{3}$ Basically, NS neurons are located in the superficial layer (laminae I-II) and respond only to noxious stimulation (high-threshold mechanical stimulation) of receptive fields, suggesting that NS neurons transmit noxious information of stimulus localization to the higher centers. ${ }^{2,3}$ Pain is known to have very complicated aspects, namely sensory-discriminative, motivational, and affective aspects. ${ }^{21}$ The sensory discriminative aspect of pain is analogous to nonnoxious sensation and thought to be involved in the discrimination of pain features such as location, intensity, and quality (via the lateral pain pathway). ${ }^{3}$ On the other hand, the motivational and affective aspects of pain are probably related to emotional and autonomic responses due to long-lasting, intense noxious stimuli (via the medial pain pathway). ${ }^{3}$ Indeed, many of the central projection neurons, such as NS neurons in the superficial layer, target for the parabrachial nucleus (PBN), ${ }^{22}$ which receives input from the NS neurons in the superficial dorsal horn and projects it to the forebrain areas such as the amygdala and hypothalamus. ${ }^{23}$ Through these connections, the spinoparabrachial pathway is likely to be involved in emotional (involving aversive) and autonomic components of pain. Therefore, it can be assumed that NS neurons are important for both localizing noxious stimulation and affecting emotional and autonomic comportments.

The present study confirmed two key findings described by us previously. ${ }^{11}$ First, we observed rapidly inhibited mechanical stimulation-induced firing of $\mathrm{SpVc}$ NS neurons by local application of DA, and second, the DA-induced effect was similar in both time course and magnitude of inhibition for $\mathrm{SpVc} \mathrm{NS}$ and WDR neurons. The sensory discriminative aspect of pain is thought to be involved in discriminating pain features, such as location, intensity, and quality, while the motivational and affective aspects of pain relate to emotional and autonomic responses due to long-lasting, intense noxious stimuli. ${ }^{21}$ The aspect is considered to be a feature of persistent pain associated, for example, with chronic inflammation or peripheral nerve injury. In the present study, we confirmed the previous observation that DA induced short-term suppression of excitability in both NS neurons and SpVc WDR neurons. ${ }^{11}$ Taken together, therefore, our findings suggested that DA inhibits not only sensory discriminative aspects of pain but also motivational and affective aspects. Further study is needed to elucidate this intriguing possibility.

\section{Mechanism underlying DA-induced hypoalgesia}

We previously showed in vivo that DA-induced motor activity is reversibly abolished by a muscarinic antagonist, implicating DA as an mAchR agonist. ${ }^{14}$ Bernardini et $\mathrm{al}^{24}$ also demonstrated using a rat skin-nerve preparation that $\mathrm{M}_{2}$ 
A

Before

DA
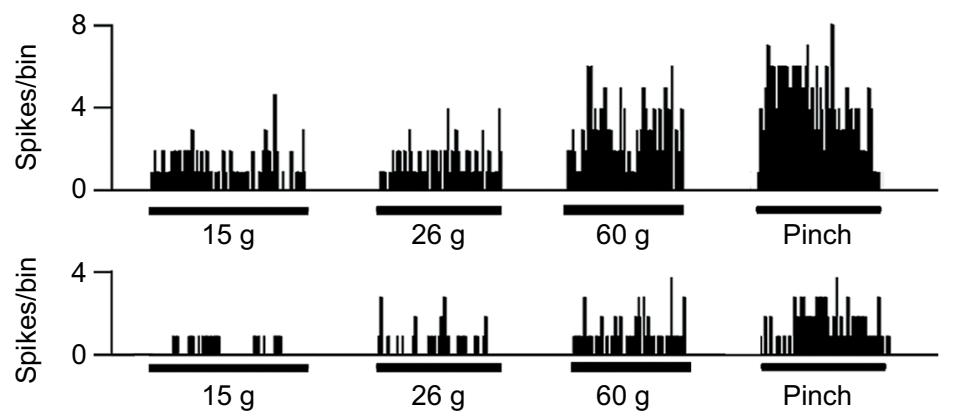

DA
+
methoctramine
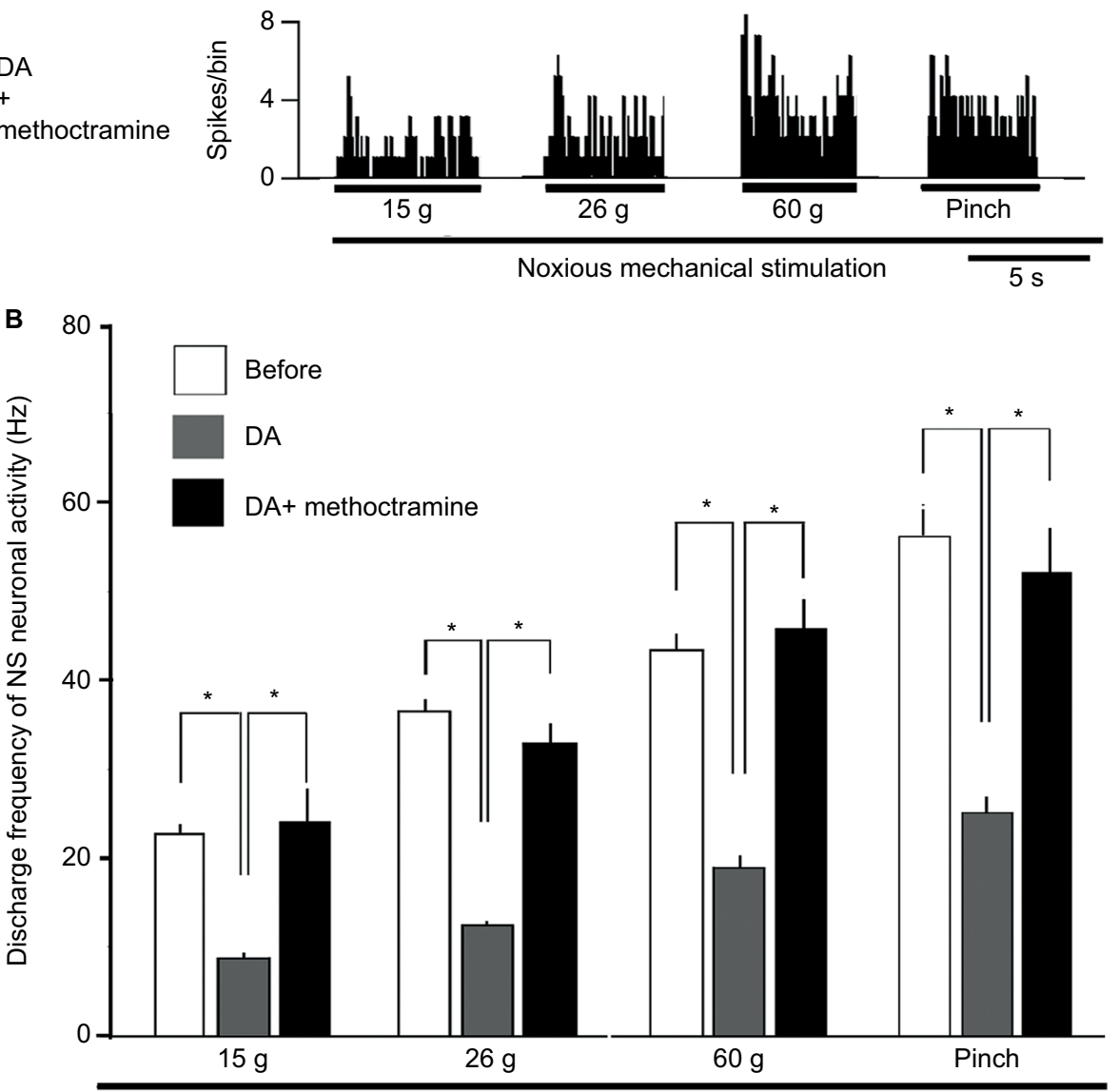

Noxious mechanical stimulation

Figure 4 Effect of pretreatment with an $M_{2} m A c h R$ antagonist on the DA-induced inhibition of SpVc NS neurons responding to noxious stimulation.

Notes: (A) Typical example of representing pretreatment with the $M_{2} m A c h R$ antagonist, methoctramine ( $\mathrm{m} M$, iv), on SpVc NS neuronal activity in response to noxious mechanical stimulation. The DA treatment-induced inhibition of SpVc NS neuronal firing in response to noxious stimulation was attenuated by pretreatment with methoctramine. (B) Summarized results showing the effects of pretreatment with methoctramine on the DA treatment-induced inhibition of SpVc NS neuronal firing in response to noxious mechanical stimulation. ${ }^{*} P<0.05$.

Abbreviations: DA, decanoic acid; iv, intravenous; mAchR, muscarinic acetylcholine receptor; NS, nociceptive specific; SpVc, spinal trigeminal nucleus caudalis.

mAchRs desensitize the peripheral terminal of C-nociceptors, possibly via the low-threshold, voltage-operated $\mathrm{K}^{+}$channels, which buildup a hyperpolarization force. ${ }^{25}$ Noguchi et $\mathrm{al}^{11}$ also demonstrated that most small-diameter TG neurons innervating facial skin expressed $\mathrm{M}_{2} \mathrm{mAchR}$ immunoreactivity $(92 \%)$, and the remaining neurons were medium-diameter TG neurons (7\%), of which 53\% also labeled positive for the neurofilament protein-200 (NF-200) myelinated fiber marker, suggesting A $\delta$-type TG neurons. On this background, the DA effects demonstrated herein, together with the result of $\mathrm{M}_{2}$ receptor antagonism, indicate that the acute administration of DA induces short-term mechanical hypoalgesia predominantly by suppressing SpVc WDR neurons via peripheral $\mathrm{M}_{2}$ receptor signaling in $\mathrm{A} \delta$ - and $\mathrm{C}$-type TG neurons.

Taken together, our results combined with the previous study ${ }^{11}$ strengthen the case for a possible mechanism underlying DA treatment-induced hypoalgesia (Figure 5), in that when noxious mechanical stimulation is applied to 
the skin, mechanosensitive ion channels open (eg, transient receptor potential [TRP] channel family, such as TRPA1), ${ }^{26}$ activating the generator potential (depolarization). This depolarization further opens voltage-dependent sodium and potassium channels, generating action potentials, which are then discharged through trigeminal primary afferent fibers (A $\delta$ - and C-fibers) to the central terminal in nociceptive $\mathrm{SpVc}$ neurons. When DA is applied (including as an original ointment) to the surface of skin, it acts on a mAchR agonist. ${ }^{14}$ Consequently, $\mathrm{M}_{2} \mathrm{mAchRs}$ have a reduced influence on the peripheral terminal of primary nociceptive neurons, ${ }^{11}$ supporting a role in affecting low-threshold voltage-operated $\mathrm{K}^{+}$ channels and the attendant hyperpolarization force. ${ }^{25}$ This effect might in turn decrease the firing frequency of action potentials in the nociceptive trigeminal nerve terminals and inhibit the conduction of pain signal to the SpVc and higher center for lateral and medial pain control (hypoalgesia).

\section{Functional significance for DA treatment- induced hypoalgesia}

CAM, such as herbal medicines and acupuncture, has been used for the treatment of clinical pain. ${ }^{4-6}$ Since polyphenolic compounds and fatty acids modulate the neuronal excitability of peripheral nerves, including the sensory system, via various voltage-dependent ionic channels, ligand-gated channels, and neurotransmitter receptors, ${ }^{10-13}$ it can be assumed that diet and dietary supplementation can potentially affect conditions associated with pain..$^{7-9}$ Thus, our results suggest that dietary

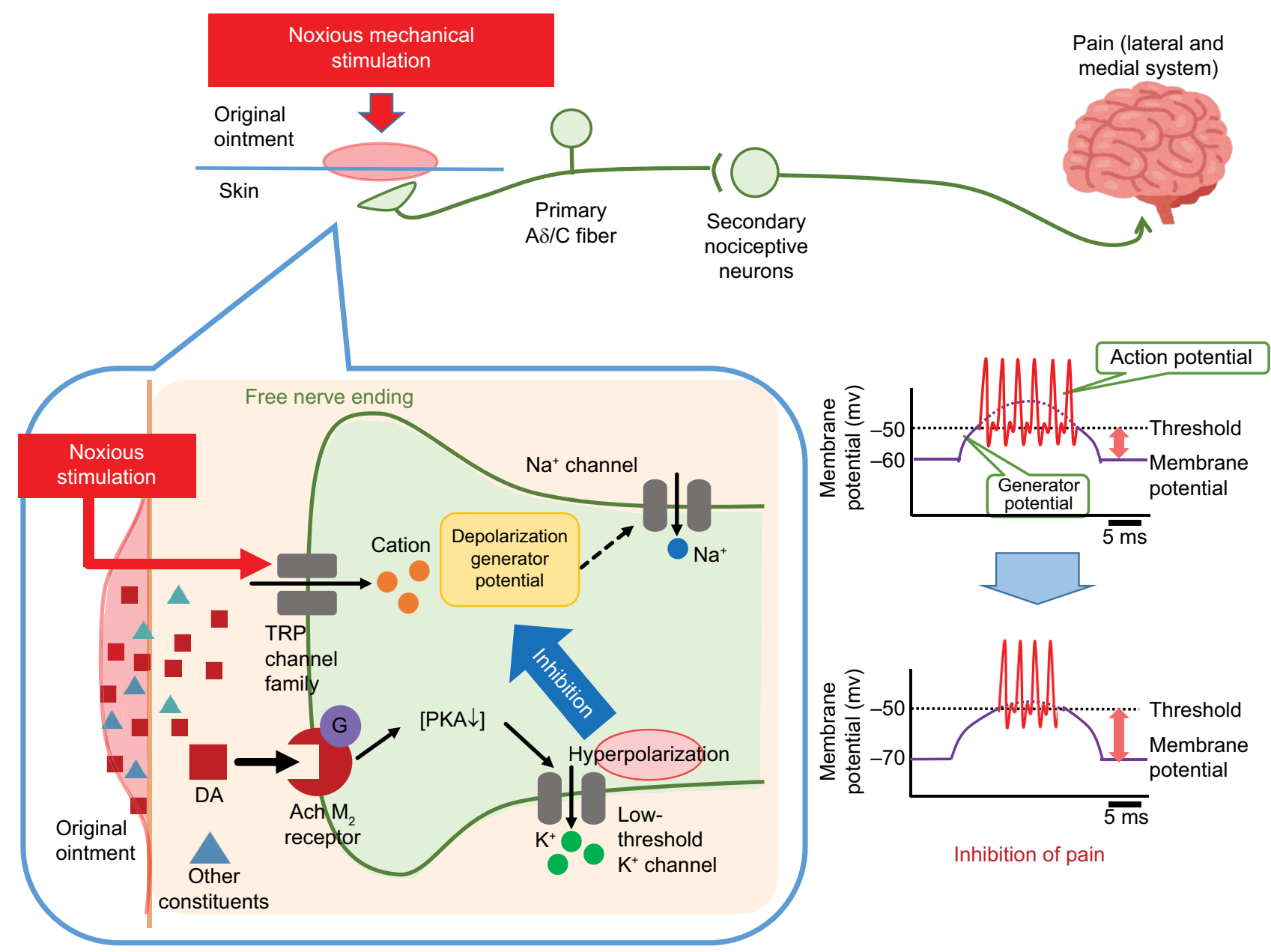

Figure 5 A possible mechanism of underlying DA treatment-induced short-term hypoalgesia.

Notes: When noxious mechanical stimulation is applied to the skin, mechanosensitive ion channels open (eg, TRP channel family, such as TRPAI), activating the generator potential (depolarization). This depolarization further opens voltage-dependent sodium and potassium channels, generating action potentials, which are in turn discharged through primary afferent fibers (A $\delta$ - and C-fibers) to the central terminal of nociceptive neurons in the SpVc. DA (including original ointment) application to the skin acts as an $m A c h R$ agonist. ${ }^{14} \mathrm{M}_{2} \mathrm{mAchRs}$ exert an inhibitory or desensitizing influence on the peripheral terminal of nociceptive neurons. This desensitization can be explained by the fact that, apart from lowering the intracellular cAMP concentration, $\mathrm{M}_{2} \mathrm{mAchRs}$ also affect low-threshold voltage-operated $\mathrm{K}^{+}$(kv) channels that buildup a hyperpolarization force. ${ }^{25}$ This effect might decrease the firing frequency of action potentials in the nociceptive nerve terminals and inhibit the conduction of pain signal to the $\mathrm{SpVc}$ and higher center for lateral and medial pain control (hypoalgesia).

Abbreviations: cAMP, cyclic adenosine monophosphate; DA, decanoic acid; PKA, protein kinase A; mAchR, muscarinic acetylcholine receptor; SpVc, spinal trigeminal nucleus caudalis; TRP, transient receptor potential. 
constituents such as DA with resveratrol, chlorogenic acid, and docosahexaenoic acids might additively contribute to the development of analgesic drugs with fewer and less severe toxic side effects for treating pathological pain, including orofacial pain. In this study, we reconfirmed the short-term hypoalgesic effect of DA application in an ointment form onto natural cutaneous tissue. ${ }^{11}$ Therefore, it can be speculated that such an ointment might effectively reduce clinical pain, such as injection-related pain during blood sampling; however, further study is needed to clarify this proposal.

\section{Conclusion}

The present study confirmed the inhibitory effect of DA on TG and SpVc NS neuronal excitability induced by short-term mechanical hypoalgesia and implicated peripheral $\mathrm{M}_{2}$ receptor signaling in mediating the DA effect. These extended in vivo findings support the potential role of DA in CAM for attenuating trigeminal nociception in the absence of confounding conditions.

\section{Acknowledgment}

The authors received no financial support for the research, authorship, or publication of this article.

\section{Author contributions}

$\mathrm{RN}, \mathrm{AU}$, and ST performed the electrophysiological and histological experiments. YA and YS interpreted the data and helped finalize the manuscript. MT participated in the design of the present study and wrote the manuscript. RN, $\mathrm{AU}$, and ST contributed equally to this work. All authors contributed to data analysis, drafting and revising the article, gave final approval of the version to be published, and agree to be accountable for all aspects of the work.

\section{Disclosure}

The authors report no conflicts of interest in this work.

\section{References}

1. Sessle BJ. Acute and chronic craniofacial pain: brainstem mechanisms of nociceptive transmission and neuroplasticity, and their clinical correlates. Crit Rev Oral Biol Med. 2000;11(1):57-91.

2. Takeda M, Matsumoto S, Sessle BJ, Shinoda M, Iwata K. Peripheral and Central Mechanisms of Trigeminal Neuropathic and Inflammatory Pain. J Oral Biosci. 2011;53(4):318-329.

3. Iwata K, Takeda M, Seog B, Shinoda M. 2017. Neurophysiology of orofacial pain. In: Farah CS, Balasubramaniam, R, McCullough, MJ. editors. Contemporary Oral Medicine. New York: Springer International Publishing AG; 2017:1-23.

4. Rao JK, Mihaliak K, Kroenke K, Bradley J, Tierney WM, Weinberger M. Use of complementary therapies for arthritis among patients of rheumatologists. Ann Intern Med. 1999;131(6):409-416.
5. Konvicka JJ, Meyer TA, McDavid AJ, Roberson CR. Complementary/ alternative medicine use among chronic pain clinic patients. J Perianesth Nurs. 2008;23(1):17-23.

6. Rosenberg EI, Genao I, Chen I, et al. Complementary and alternative medicine use by primary care patients with chronic pain. Pain Med. 2008;9(8):1065-1072.

7. Shir Y, Raja SN, Weissman CS, Campbell JN, Seltzer Z. Consumption of soy diet before nerve injury preempts the development of neuropathic pain in rats. Anesthesiology. 2001;95(5):1238-1244.

8. Ernst E. Complementary medicine. Curr Opin Rheumatol. 2003;15(2): 151-155.

9. Tall JM, Raja SN. Dietary constituents as novel therapies for pain. Clin J Pain. 2004;20(1):19-26.

10. Shimazu Y, Shibuya E, Takehana S, et al. Local administration of resveratrol inhibits excitability of nociceptive wide-dynamic range neurons in rat trigeminal spinal nucleus caudalis. Brain Res Bull. 2016;124: 262-268.

11. Noguchi Y, Matsuzawa N, Akama Y, et al. Dietary constituent, decanoic acid suppresses the excitability of nociceptive trigeminal neuronal activity associated with hypoalgesia via muscarinic M2 receptor signaling. Mol Pain. 2017;13:1744806917710779.

12. Kakita K, Tsubouchi H, Adachi M, Takehana S, Shimazu Y, Takeda M. Local subcutaneous injection of chlorogenic acid inhibits the nociceptive trigeminal spinal nucleus caudalis neurons in rats. Neurosci Res. 2018;134:49-55.

13. Mitome K, Takehana S, Oshima K, Shimazu Y, Takeda M. Local anesthetic effect of docosahexaenoic acid on the nociceptive jaw-opening reflex in rats. Neurosci Res. Epub 2018 Feb 23.

14. Gwynne RM, Thomas EA, Goh SM, Sjövall H, Bornstein JC. Segmentation induced by intraluminal fatty acid in isolated guinea-pig duodenum and jejunum. J Physiol. 2004;556(Pt 2):557-569.

15. Zimmermann M. Ethical guidelines for investigations of experimental pain in conscious animals. Pain. 1983;16(2):109-110.

16. Paxinos G, Watson C. The Rat Brain in Stereotaxic Coordinates. 2nd ed. New York, NY: Academic Press; 1986.

17. Sekiguchi K, Takehana S, Shibuya E, et al. Resveratrol attenuates inflammation-induced hyperexcitability of trigeminal spinal nucleus caudalis neurons associated with hyperalgesia in rats. Mol Pain. 2016;12(12):1744806916643082.

18. Takeda M, Tanimoto T, Nasu M, Ikeda M, Kadoi J, Matsumoto S. Activation of NK1 receptor of trigeminal root ganglion via substance $\mathrm{P}$ paracrine mechanism contributes to the mechanical allodynia in the temporomandibular joint inflammation in rats. Pain. 2005;116(3):375-385.

19. Takeda M, Takahashi M, Matsumoto S. Contribution of activated interleukin receptors in trigeminal ganglion neurons to hyperalgesia via satellite glial interleukin-1beta paracrine mechanism. Brain Behav Immun. 2008;22(7):1016-1023.

20. Gregg JM, Dixon AD. Somatotopic organization of the trigeminal ganglion in the rat. Arch Oral Biol. 1973;18(4):487-498.

21. Dubner R. The effect of behavioral state on the sensory processing of nociceptive and non-nociceptive information. Prog Brain Res. 1988;77:213-228.

22. Todd AJ. Neuronal circuitry for pain processing in the dorsal horn. Nat Rev Neurosci. 2010;12:823-836.

23. Gauriau C, Bernard JF. Pain pathways and parabrachial circuits in the rat. Exp Physiol. 2002;87(2):251-258.

24. Bernardini N, Sauer SK, Haberberger R, Fischer MJ, Reeh PW. Excitatory nicotinic and desensitizing muscarinic (M2) effects on C-nociceptors in isolated rat skin. J Neurosci. 2001;21(9):3295-3302.

25. Pan ZZ, Williams JT. Muscarine hyperpolarizes a subpopulation of neurons by activating an M2 muscarinic receptor in rat nucleus raphe magnus in vitro. J Neurosci. 1994;14(3 Pt 1):1332-1338.

26. Kwan KY, Glazer JM, Corey DP, Rice FL, Stucky CL. TRPA1 modulates mechanotransduction in cutaneous sensory neurons. J Neurosci. 2009;29(15):4808-4819. 
The Journal of Pain Research is an international, peer reviewed, open access, online journal that welcomes laboratory and clinical findings in the fields of pain research and the prevention and management of pain. Original research, reviews, symposium reports, hypothesis formation and commentaries are all considered for publication.
The manuscript management system is completely online and includes a very quick and fair peer-review system, which is all easy to use. Visit http://www.dovepress.com/testimonials.php to read real quotes from published authors. 